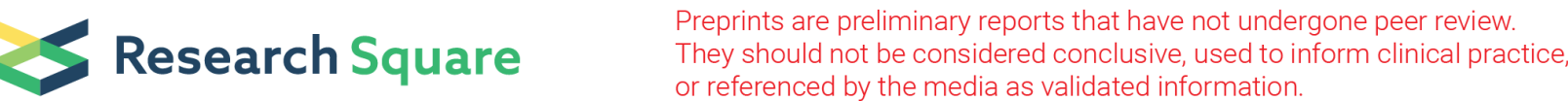

\section{LncRNA MIR4435-2HG Promotes Proliferation, Migration, Invasion and EMT Via Targeting miR-22- 3p/TMEM9B in Breast Cancer}

Jing Ke

Affiliated Hospital of Nantong University

Quhui Wang

Affiliated Hospital of Nantong University

Wei Zhang

Affiliated Hospital of Nantong University

Haijun Mei ( 15996692626@163.com )

Affiliated Hospital of Nantong University

Research Article

Keywords: Breast cancer, MIR4435-2HG, MiR-22-3p, TMEM9B

Posted Date: September 29th, 2021

DOI: https://doi.org/10.21203/rs.3.rs-860533/v1

License: (c) (i) This work is licensed under a Creative Commons Attribution 4.0 International License.

Read Full License 


\section{Abstract}

Background: Breast cancer, as the malignancy with the highest incidence rate and mortality rate in women, seriously threatens human life and health. Pieces of evidence have suggested that long noncoding RNAs (IncRNAs) possess important effects on regulating the occurrence and development of breast cancer.

Results: In the present study, MIR4435-2HG was highly expressed in breast cancer tissues and cells. Down-regulation of MIR4435-2HG inhibited the viability, proliferation, migration, invasion and epithelialmesenchymal transition (EMT) of breast cancer cell lines by Cell Counting Kit-8 (CCK-8), colony formation, transwell migration and invasion, immunofluorescence and western blot assays. Dualluciferase reporter assay and RNA pull-down analysis confirmed that miR-22-3p was a target of MIR44352HG. Over-expression of MicroRNA-22-3p (miR-22-3p) obviously inhibited the viability, proliferation, migration, invasion and EMT of breast cancer cell lines. Transmembrane protein 9 domain family member B (TMEM9B) was up-regulated in breast cancer tissues and cell lines. Dual-luciferase reporter assay confirmed that TMEM9B was a target of miR-22-3p. TMEM9B inhibition partially restored the effects of MIR4435-2HG/miR-22-3p on the viability, proliferation, migration, invasion and EMT of breast cancer cell lines.

Conclusions: MIR4435-2HG potentially played a tumor-promoting role in the occurrence and development of breast cancer, which might be achieved by regulating the miR-22-3p/TMEM9B axis.

\section{Introduction}

Breast cancer is a kind of malignant tumor originated from breast epithelial tissues and is also the most common malignant disease in female patients [1]. Recent reports show that the incidence rate of breast cancer is increasing annually [2]. There are more than 1.3 million newly diagnosed cases of breast cancer in the world every year, and in China, breast cancer has accounted for $15 \%$ of new female tumors [3]. With the development of early detection, early diagnosis, early treatment, and prognosis monitoring of breast cancer, the overall mortality of breast cancer patients has decreased significantly [4]. However, malignant proliferation, recurrence, metastasis and other factors may compensate these benefits. There are more than 45,000 deaths of breast cancer patients in the world every year [5]. China accounts for $9.2 \%$ of the global deaths and is the greatest contributor [6]. Therefore, breast cancer is still a serious threat to women's life and health in China. Further understanding of the molecular mechanisms of the occurrence and development of breast cancer and finding the key molecules that can effectively inhibit the proliferation, invasion and metastasis of breast cancer cells will be conducive to the targeted treatment of clinical breast cancer $[7,8]$.

Long non-coding RNAs (LncRNA) are a kind of non-coding RNAs with a length from $200 \mathrm{bp}$ to $100 \mathrm{kbp}$. LncRNAs account for $4 \%-9 \%$ of the transcripts in the mammalian genome and gained increasing interest [9]. With the rapid development of RNA sequencing, epigenome technology and computational prediction 
technology, an increasing number of IncRNAs have been found. Previous studies have shown that IncRNAs are abnormally expressed in breast cancer. Yang et al. identify more than 1300 abnormal IncRNAs in breast cancer by sequencing [10]. In addition, Shen et al. identify more than 1750 IncRNAs differentially expressed in triple-negative breast cancer [11]. These results suggest that abnormal expression of IncRNAs may play an important role in the carcinogenesis of breast cancer. Lv et al. find that the expressions of IncRNAs in triple-negative breast cancer are different from those in non-triple negative breast cancer, which can be used as biomarkers for individual diagnosis, and may also be potential targets for evaluating individual therapeutic efficacy [12]. Moreover, IncRNA H19, SRA, Isinct5 and UCA1 promote the proliferation and/or metastasis of breast cancer. On the contrary, IncRNA GAS5 and XIST inhibit the proliferation and/or metastasis of breast cancer [13].

LncRNA MIR4435-2HG, known as AK001796 and LINC00978, is encoded on human chromosome 2q13 and has been regarded as a new oncogenic IncRNA in many types of cancer [14]. For example, a high level of MIR4435-2HG is detected in colorectal cancer tissues [15]. Increased level of MIR4435-2HG is significantly correlated with TNM stage and carcinoembryonic antigen level before treatment. High MIR4435-2HG expression has a poorer progression-free survival and overall survival (OS) rate [15]. In addition, MIR4435-2HG is up-regulated in hepatocellular carcinoma tissues, and the expression of MIR4435-2HG is significantly affected by tumor size instead of tumor metastasis [16]. Additionally, MIR4435-2HG is over-expressed in ovarian cancer tissues and cells. It is reported that MIR4435-2HG expression is negatively related to the health conditions of ovarian cancer patients. MIR4435-2HG knockdown inhibits proliferation, invasion and migration but induces apoptosis of ovarian cancer cells via the miR-128-3p/CDK14 axis [17]. However, the exact functions and corresponding mechanisms of MIR4435-2HG in the occurrence and development of breast cancer have been rarely reported [18-20]. Therefore, the present study was designed to determine whether MIR4435-2HG exerted its functional role in the growth and metastasis of breast cancer and investigate the possible mechanisms.

\section{Materials And Methods \\ 2.1 Clinical tissue collection}

A total of 60 tissues, including 15 tissues in stage I + II and 15 tissues in stage III + IV, and 30 corresponding adjacent tissues were collected from Affiliated Hospital of Nantong University. All patients did not receive any chemotherapy and signed written informed consent. Moreover, the experimental procedures were approved by the Ethics Committee of Affiliated Hospital of Nantong University.

\subsection{Cell lines}

Breast cancer cell lines, including Hs-578T, MCF-7, ZR-75-30 and HCC1937, and human breast epithelial cell line MCF-10A were obtained from the Chinese Academy of Science (Shanghai, China). Cells were routinely cultured in Dulbecco's Modified Eagle Medium (DMEM, KeyGEN, Nanjing, China) supplemented 
with $10 \%$ fetal bovine serum (FBS, Gibco, USA) and 1\% penicillin-streptomycin (Sigma-Aldrich, USA) in a humidified atmosphere with $5 \% \mathrm{CO}_{2}$ at $37^{\circ} \mathrm{C}$.

\subsection{Cell transfection}

Short hairpin RNA (sh)-MIR4435-2HG, sh-TMEM9B, miR-22-3p mimic, miR-22-3p inhibitor and corresponding negative control (NC) vectors were obtained from Gene Pharma Company (Shanghai, China). Lipofectamine 3000 (Invitrogen, USA) was performed for cell transfection based on the specification.

\subsection{Cell Counting Kit-8 (CCK-8) assay}

Transfected MCF-7 and ZR-75-30 cells at the density of $1 \times 10^{4}$ cells/well were maintained in 96-well plates and cultured for different time points $(0,24,48$ and $72 \mathrm{~h}$, respectively). Then, cell viability was determined using a CCK-8 kit (Beyotime, Shanghai, China) according to the protocols of the manufacturer. The optical density was measured by the OD value at $450 \mathrm{~nm}$ by using a microplate reader (BioTek Instruments Inc., Winooski, VT, USA).

\subsection{Colony formation assay}

Transfected MCF-7 and ZR-75-30 cells $\left(1 \times 10^{3}\right.$ cells/well) were seeded in six-well plates. The media was replaced with a fresh culture medium every 2-3 days for 2 weeks. Subsequently, MCF-7 and ZR-75-30 cells were stained with $10 \%$ crystal violet for 30 min and observed by using a microscope (Olympus, Tokyo, Japan).

\subsection{Wound healing assay}

Transfected MCF-7 and ZR-75-30 cells $\left(5 \times 10^{5}\right.$ cells/well) were seeded into a six-well plate. Once the cells were $100 \%$ confluent, a wound was made on the surface of the cell using a $200-\mu \mathrm{L}$ tip, and a serum-free medium was used. After 0 and 48 h, MCF-7 and ZR-75-30 cells were observed under an inverted microscope (Tokyo, Japan), and the distance between the wounds was recorded.

\subsection{Transwell migration and invasion assays}

For migration and invasion assays, transfected MCF-7 and ZR-75-30 cells $\left(1 \times 10^{6}\right.$ cells/well) were seeded in the transwell chambers containing an $8 \mu \mathrm{m}$ size porous membrane (Corning, NY, USA). The upper chamber was inserted without or with matrigel, while the lower chamber was added by $20 \%$ FBS. After $48 \mathrm{~h}$ incubation at $37^{\circ} \mathrm{C}$, the non-migrating or invading cells in the upper chamber were removed by cotton wool. The migrated or invaded cells in the upper chamber were stained with $0.1 \%$ crystal violet solution (Sangon Biotech, Shanghai, China) and counted using a microscope (Olympus, Tokyo, Japan).

\subsection{RNA extraction and qRT-PCR analysis}

RNA from clinical tissues and cell lines was extracted according to the instructions of the TRIzol reagent, and the $\mathrm{OD}_{260} / \mathrm{OD}_{280}$ of the RNA solution was also detected. The reverse transcription reaction was 
conducted according to the instructions of the cDNA synthesis kit, and the PCR reaction solution was configured according to the instructions of the Taq enzyme mixture. The amplification program was set up for primer sequence as listed below, and a PCR amplification reaction was conducted. PCR reaction conditions: $50^{\circ} \mathrm{C}$ for $2 \mathrm{~min}, 95^{\circ} \mathrm{C}$ for $10 \mathrm{~min}(1 \mathrm{cycle}), 9^{\circ} \mathrm{C}$ for $5 \mathrm{~s}, 65^{\circ} \mathrm{C}$ for $1 \mathrm{~min}, 75^{\circ} \mathrm{C}$ for $20 \mathrm{~s}(45$ cycles); and $75^{\circ} \mathrm{C}$ for $5 \mathrm{~min}$ ( 1 cycle). Each real-time PCR was repeated 3 times. MIR4435-2HG forward, $5^{\prime}$ CGGAGCATGGAACTCGACA-3', and reverse, 5'-CAAGTC TCACACATCCGGG-3'. MiR-22-3p forward, 5'AAGCTGCCAGTTGAAGAACTGTA-3', and reverse, 5'-GCTGTCAACGATACGCTACGTAAC-3'. TMEM9B forward, 5'-AAAGTCCGCCATTT TGCCAC-3', and reverse, 5'-ATTCGGGGCTCTGTAGTCCT-3'. U6 forward, 5'CTCGCTTCGGCA GCACA-3', and reverse, 5'-AACGCTTCACGAATTTGCGT-3'. $\beta$-actin forward, 5'TCGTGGAAGG ACTCATGACC-3', and reverse, 5'-ATGATGTTCTGGAGAGCCCC-3'. The fold change in gene expression was calculated using the $2^{-\triangle \triangle C T}$ method after normalizing to the expression level of $U 6$ and $\beta$ actin.

\subsection{Western blot assay}

Protein from breast cancer cell lines was isolated using RIPA lysis buffer and quantified with a BCA kit (Beyotime, Shanghai, China). Protein was separated via 12\% SDS-PAGE and then transferred onto PVDF membranes (EMD Millipore, USA). Subsequently, the membranes were blocked with $5 \%$ skimmed milk and treated with primary antibodies overnight at $4^{\circ} \mathrm{C}$. Membranes were washed and probed with HRPconjugated secondary antibody $(1: 2,000$, ab6728, Abcam, USA) for $1 \mathrm{~h}$ at room temperature. Protein bands were visualized by enhanced chemiluminescence (EMD Millipore) and quantified using ImageJ software (version 4.3; National Institutes of Health). The primary antibodies used (all from Abcam) were as follows: Anti-E-cadherin (1: 1, 000, ab1416), anti-vimentin (1: 1, 000, ab92547), anti-a-smooth muscle actin (SMA, 1: 1, 000, ab32575), anti-TMEM9B (1: 1, 000, ab189253) and anti- $\beta$-actin (1: 1, 000, ab8227).

\subsection{Immunofluorescence assay}

Transfected MCF-7 and ZR-75-30 cells were cultured in six-well plates at a density of $1 \times 10^{5}$ cells/well. After fixing in $4 \%$ paraformaldehyde and permeabilization with $0.2 \%$ Triton X-100 in PBS, cells were treated with primary antibodies against a-SMA $\left(1: 1,000\right.$, ab32575) overnight at $4^{\circ} \mathrm{C}$. Subsequently, cells were incubated with goat anti-rabbit IgG H\&L secondary antibody (1: 1, 000, ab150077). The nuclei were counter-stained with DAPI. Positive staining was observed under a fluorescence microscope (Zeiss AG, Germany).

\subsection{Subcellular fractionation analysis}

Cytoplasmic and nuclear fractions were extracted from MCF-7 and ZR-75-30 cells using the NE-PER Nuclear and Cytoplasmic Extraction reagent (Thermo Fisher Scientific, Inc. USA). RNA from each fraction was measured by qRT-PCR. U6 was used as internal references for MIR4435-2HG in cytoplasmic and nuclear fractions, respectively.

\subsection{RNA pull-down analysis}


MCF-7 and ZR-75-30 cells were incubated with biotin-labeled miR-22-3p-wild-type (WT) or -mutant (Mut) for $48 \mathrm{~h}$ and lysed in the specific buffer. The lysate was incubated with magnetic beads and washed three times with precooled lysis buffer and salt buffer solution. Finally, the bound RNA was purified with TRIzol®. MIR4435-2HG or miR-22-3p enrichment was analyzed using qRT-PCR.

\subsection{Dual-luciferase reporter assay}

The sequence of MIR4435-2HG-WT and Transmembrane protein 9 domain family member B (TMEM9B)WT and MIR4435-2HG-Mut and TMEM9B-Mut were inserted into the pmirGLO reporter vector (GenScript, Shanghai, China). Subsequently, pmirGLO-MIR4435-2HG-WT/Mut and pmirGLO-TMEM9B-WT/Mut were treated with miR-22-3p mimic or NC into MCF-7 and ZR-75-30 cells using Lipofectamine 3000 (Invitrogen; Thermo Fisher Scientific, Inc., USA). Luciferase activity was measured using a dual-luciferase reporter assay system (Promega Corporation, USA).

\subsection{Statistical analysis}

GraphPad Prism 6.0 software was used for graphing. All data were expressed as mean \pm standard deviation (SD). SPSS 17.0 software was used for statistical analysis. Data comparison between groups was performed by analysis of variance. A pairwise comparison between means was performed by $t$ test. $P<0.05$ was considered statistically significant.

\section{Results}

\subsection{MIR4435-2HG is highly expressed in breast cancer tissues and cell lines}

To investigate the possible role and underlying mechanisms of MIR4435-2H in breast cancer, the expression of MIR4435-2H in breast cancer tissues was firstly evaluated by qRT-PCR. The data of Fig. $1 \mathrm{~A}$ showed that MIR4435-2H was significantly over-expressed in breast cancer tissues compared with nontumor tissues. In addition, the expression of MIR4435-2H in breast cancer patients at different TNM stages was also analyzed. The data of Fig. 1B revealed that MIR4435-2H expression was higher in patients at advanced stage III + IV than that in patients at stage I+ II. Moreover, the clinical significance of aberrant MIR4435-2H expression in the prognosis of patients was further investigated. The results in Fig. 1C indicated that patients with low MIR4435-2H expression exhibited improved percent survival compared with patients with high MIR4435-2H expression. Furthermore, qRT-PCR was performed to detect the expression of MIR4435-2H in breast cancer cells. As expected, the data of Fig. 1D showed that compared with MCF-10A cells, the expression of MIR4435-2H was obviously higher in breast cancer cells, especially in MCF-7 and ZR-75-30 cells. These data suggested that MIR4435-2H was up-regulated in breast cancer tissues and cell lines and might function as an oncogene in the occurrence and development of breast cancer. 


\subsection{Down-regulation of MIR4435-2H inhibits the viability, proliferation, migration, invasion and EMT of breast cancer cells}

To determine the potential role of MIR4435-2H in breast cancer, MCF-7 and ZR-75-30 cells were transfected with sh-MIR4435-2H, and transfection efficiency was detected by the qRT-PCR assay. As shown in Fig. 2A, the expression of MIR4435-2H was obviously decreased in MCF-7 and ZR-75-30 cells transfected with sh-MIR4435-2H. Then, a CCK-8 assay was used to evaluate the effects of MIR4435-2H on the viability of MCF-7 and ZR-75-30 cells, and the data of Fig. 2B showed that down-regulation of MIR4435-2H significantly inhibited the viability of MCF-7 and ZR-75-30 cells in a time-dependent manner. Besides, a colony formation assay was performed to assess the effects of MIR4435-2H on the proliferation of MCF-7 and ZR-75-30 cells. The data in Fig. 2C showed that MIR4435-2H inhibition significantly suppressed the proliferation of MCF-7 and ZR-75-30 cells. Moreover, the role of MIR4435-2H in metastasis of MCF-7 and ZR-75-30 cells was also investigated. The data of wound healing and transwell results showed that down-regulation of MIR4435-2H significantly inhibited migration and invasion of MCF-7 and ZR-75-30 cells displayed in Fig. 2D and 2E. Furthermore, the effects of MIR4435$2 \mathrm{H}$ on the EMT progress of MCF-7 and ZR-75-30 cells were explored. The data of immunofluorescence assay showed that sh-MIR4435-2H significantly inhibited a-SMA expression (Fig. 2F). Western blotting was performed to evaluate the effects of MIR4435-2H on the expression levels of EMT-related proteins, including E-cadherin, vimentin and a-SMA. The data of Fig. $2 \mathrm{G}$ revealed that sh-MIR4435-2H significantly decreased the protein expressions of Vimentin and a-SMA and increased the level of E-cadherin protein in MCF-7 and ZR-75-30 cells. These data suggested that down-regulation of MIR4435-2H inhibited the viability, proliferation, migration, invasion and EMT of breast cancer cells.

\subsection{MIR4435-2H acts as a competing endogenous RNA (ceRNA) via sponging miR-22-3p}

To determine the possible mechanisms of MIR4435-2H in breast cancer, the distribution of MIR4435-2H was initially investigated by subcellular fraction analysis. As shown in Fig. 3A, MIR4435-2H was primarily found in the cytoplasm. In addition, to verify whether MIR4435-2H acted as a ceRNA, bioinformatics tools were utilized to acquire miRNAs that potentially bound to MIR4435-2H. As indicated in Fig. 3B, miR-22-3p was predicted to possess the target domain for MIR4435-2H. Then miR-22-3p mimic and mimic NC were transfected into MCF-7 and ZR-75-30 cells, and qRT-PCR was performed to detect transfection efficiency (Fig. 3C). Dual-luciferase reporter analysis was performed to validate the interaction between MIR4435$2 \mathrm{H}$ and miR-22-3p. As expected, the data of Fig. 3D showed that exogenous expression of miR-22-3p significantly decreased the luciferase intensity of MCF-7 and ZR-75-30 cells transfected with MIR44352H-WT. Similarly, RNA pull-down data showed that MIR4435-2H was highly enriched in MCF-7 and ZR-7530 cells treated with miR-22-3p-WT (Fig. 3E). Furthermore, the expression of miR-22-3p was downregulated in breast cancer tissues and cell lines (Fig. 3F and $3 \mathrm{G}$ ) and was significantly up-regulated in 
MCF-7 and ZR-75-30 cells transfected with sh-MIR4435-2H (Fig. 3H). Finally, a negative association was also discovered between MIR4435-2H and miR-22-3p levels in breast cancer tissues (Fig. 3I). These data suggested that MIR4435-2H served as a ceRNA for miR-22-3p in breast cancer.

\subsection{Up-regulation of miR-22-3p inhibits the viability, proliferation, migration, invasion and EMT of breast cancer cells}

CCK-8 assay was performed to evaluate the effects of miR-22-3p on the viability of MCF-7 and ZR-75-30 cells. As shown in Fig. 4A, miR-22-3p mimic significantly inhibited the viability of MCF-7 and ZR-75-30 cells in a time-dependent manner. Moreover, a colony formation assay was performed to assess the effects of miR-22-3p on the proliferation of MCF-7 and ZR-75-30 cells. The data in Fig. 4B showed that up-regulation of miR-22-3p significantly suppressed the proliferation of MCF-7 and ZR-75-30 cells. Then the data of wound healing and transwell assays showed that miR-22-3p mimic significantly inhibited the migration and invasion of MCF-7 and ZR-75-30 cells displayed in Fig. 4C and 4D. Additionally, a immunofluorescence assay was performed to evaluate the effects of miR-22-3p on the expression of aSMA. The results showed that miR-22-3p mimic significantly inhibited a-SMA expression (Fig. 4E). Furthermore, western blotting was performed to evaluate the effects of miR-22-3p on the expression levels of EMT-related proteins. The data in Fig. 4F revealed that miR-22-3p mimic significantly decreased the protein expressions of Vimentin and a-SMA and increased the level of E-cadherin protein in MCF-7 and ZR-75-30 cells. These data suggested that up-regulation of miR-22-3p inhibited the viability, proliferation, migration, invasion and EMT of breast cancer cells.

\subsection{TMEM9B acts as a direct target of miR-22-3p}

To investigate the downstream targets of miR-22-3p, bioinformatics tools were utilized to acquire mRNAs potentially bound to miR-22-3p. As indicated in Fig. 5A, TMEM9B was predicted to possess the target domain for miR-22-3p. Dual-luciferase assay results further confirmed the target relationship between miR-22-3p and TMEM9B (Fig. 5B). In addition, qRT-PCR was performed to detect TMEM9B expression in breast cancer tissues and cell lines. As shown in Fig. 5C and 5D, TMEM9B was highly expressed in breast cancer tissues and cell lines. Furthermore, qRT-PCR and western blot assays were performed to evaluate the mRNA and protein levels of TMEM9B in MCF-7 and ZR-75-30 cells transfected with miR-22-3p mimic or NC mimic. The data in Fig. 5E and 5F revealed that the mRNA and protein expressions of TMEM9B were significantly decreased in MCF-7 and ZR-75-30 cells transfected with miR-22-3p mimic. Finally, a negative association was discovered between miR-22-3p and TMEM9B levels in breast cancer tissues (Fig. 5G). These data suggested that TMEM9B was a direct target of miR-22-3p and was negatively associated with miR-22-3p in breast cancer.

3.6 MIR4435-2H regulates the viability, proliferation, migration, invasion and EMT of breast cancer cells by modulating the miR-22-3p/TMEM9B axis 
To further determine whether MIR4435-2H exhibited its role by regulating the miR-22-3p/TMEM9B axis, a series of rescue assays were performed. Firstly, CCK-8 and colony formation assays were performed. As shown in Fig. 6A and 6B, MIR4435-2H knockdown inhibited the viability and proliferation of MCF-7 and ZR-75-30 cells. However, the miR-22-3p inhibitor promoted MCF-7 and ZR-75-30 cell viability and proliferation, while TMEM9B2 inhibition exhibited inhibitory effects on the viability and proliferation of MCF-7 and ZR-75-30 cells. Moreover, wound healing and transwell assays were performed, and the data indicated that MIR4435-2H knockdown inhibited the migration and invasion of MCF-7 and ZR-75-30 cells. However, miR-22-3p inhibitor promoted MCF-7 and ZR-75-30 cell migration and invasion, while TMEM9B2 inhibition exhibited suppressive effects on the migration and invasion of MCF-7 and ZR-75-30 cells (Fig. 6C and 6D). Immunofluorescence and western blot assays were performed, and the data in Fig. 6E and $6 \mathrm{~F}$ illustrated that MIR4435-2H knockdown inhibited the EMT progression of MCF-7 and ZR-75-30 cells. However, miR-22-3p inhibitor promoted MCF-7 and ZR-75-30 cell EMT progression, while TMEM9B2 inhibition exhibited inhibitory effects on EMT progression of MCF-7 and ZR-75-30 cells. These data suggested MIR4435-2H exerted its roles by modulating the miR-22-3p/TMEM9B axis.

\section{Discussion}

A large number of studies have confirmed that abnormal expression of MIR4435-2HG plays an essential role in multiple tumors, including gastric cancer, prostate carcinoma, and oral squamous cell carcinoma [21-24]. In this manuscript, MIR4435-2HG was significantly up-regulated in breast cancer tissues and cell lines. In addition, breast cancer patients with low MIR4435-2HG expression exhibited improved percent survival compared with those with high MIR4435-2HG expression, which is consistent with previous studies [15-17], suggesting that MIR4435-2HG may act as an oncogene in the occurrence and progression of breast cancer. Functionally, our data showed that down-regulation of MIR4435-2HG notably inhibited the viability, proliferation, migration, invasion and EMT progress of breast cancer cells. Similarly, MIR4435-2HG exhibited its role in promoting the proliferation and metastasis of colorectal cancer and ovarian cancer [25-27]. These data suggested that MIR4435-2HG might act as an oncogene in the occurrence and progression of breast cancer.

LncRNAs affect the occurrence and development of tumors via post-transcriptional regulation [28]. Multiple IncRNAs may regulate gene expression, thus reducing the number of available miRNAs in cells [29]. Therefore, IncRNAs act as competing ceRNAs to modulate the expression of the target gene via sponging miRNAs [30]. MIR4435-2HG has been reported to exert ceRNA function in osteoarthritis, glioma, hepatocellular carcinoma and lung cancer [31, 32]. In this manuscript, bioinformatic analysis of MIR4435$2 \mathrm{HG}$-miRNA prediction was performed through online software, and the target relationship was verified by a dual-luciferase reporter and pull-down assays. All the data indicated that miR-22-3p was a direct target of MIR4435-2HG. Previous studies have illustrated that miR-22-3p exerted essential effects on the development of many tumors, including bladder cancer, gastric cancer, and lung cancer [33-36]. The data showed that the level of miR-22-3p was dramatically reduced in breast cancer tissues and cell lines, which was inversely correlated with MIR4435-2HG expression in breast cancer tissues. Moreover, up- 
regulation of miR-22-3p inhibited the viability, proliferation, migration, invasion and EMT progress of breast cancer cells. These results indicated that miR-22-3p acted as a tumor suppressor in breast cancer.

MiRNAs act as tumor suppressors through the restraint of their target genes to participate in breast cancer progression [37]. In this study, TMEM9B was confirmed as a target of miR-22-3p containing the putative miRNA response sequences within its 3'-UTR by starBase v2.0. Tmem9B, as a glycosylated protein located on the lysosomal membrane, has been previously discovered as an NF-KB inducer in large-scale cDNA over-expression screens [38]. Furthermore, TMEM9B is a key component of inflammatory signaling pathways [39]. A remarkable negative correlation between the expression levels of TMEM9B and miR-22-3p in breast cancer tissues was found in this study. TMEM9B was a direct target of miR-22-3p in breast cancer cells, which indicated that the ceRNA system existed among MIR4435-2HG, miR-22-3p and TMEM9B in breast cancer. Next, whether MIR4435-2HG/miR-22-3p/TMEM9B axis conduced to the progression of breast cancer in vitro was further explored. The relationship between MIR4435-2HG/miR-22-3P/TMEM9B axis and the progression of breast cancer was investigated in vitro. The data presented that the effects of MIR4435-2HG knockdown reduced TMEM9B expression level by the promotion of miR-22-3p. TMEM9B inhibition partly restored the effects of the miR-22-3p inhibitor on the viability, proliferation, migration, invasion and EMT progression of breast cancer cells transfected with sh-MIR4435-2HG.

To conclude, MIR4435-2HG was regarded as a vital mediator of cell growth and metastasis in breast cancer. MIR4435-2HG was obviously up-regulated in breast cancer tissues and cell lines, and MIR4435-

$2 \mathrm{HG}$ knockdown inhibited breast cancer progression by acting as a ceRNA to down-regulate TMEM9B through competitively binding to miR-22-3p. These results indicated that MIR4435-2HG/miR-223p/TMEM9B axis might be a potential therapeutic basis for the treatment of breast cancer.

\section{Declarations}

\section{Conflicts of interest}

The authors state that there are no conflicts of interest.

\section{Ethics approval and consent to participate}

The analysis was performed according to the principles of Helsinki Declaration. Written informed consent was obtained from all patients. The experimental procedures were approved by the Ethics Committee of Affiliated Hospital of Nantong University.

\section{Consent for publication}

The authors give consent to the publication in the journal.

\section{Availability of data and material}


All data generated or analysed during this study are included in this published article.

\section{Funding}

This work was supported by Nantong Municipal Science and Technology Plan(MSZ19210)

\section{Authors' contributions}

Jing Ke and Haijun Mei conceived and designed the study. Quhui Wang and Wei Zhang conducted most of the experiments. Wei Zhang analyzed the data. Quhui Wang performed the literature search and data extraction. Jing Ke drafted the manuscript. Jing Ke and Haijun Mei finalized the manuscript. All authors read and approved the final manuscript.

\section{Acknowledgements}

We deeply appreciate the supports by all participants.

\section{References}

1. Noguchi E, Shien T, Iwata H. Current status of PD-1/PD-L1 blockade immunotherapy in breast cancer. Jpn J Clin Oncol, 2021, 51 (3): 321-332.

2. Sopik V. International variation in breast cancer incidence and mortality in young women.Breast Cancer Res Treat, 2021, 186 (2): 497-507.

3. Xie Y, Valdimarsdóttir UA, Wang C, Zhong X, Gou Q, Zheng H, Deng L, He P, Hu K, Fall K, Fang F, Tamimi RM, Luo T, Lu D. Public health insurance and cancer-specific mortality risk among patients with breast cancer: A prospective cohort study in China. Int J Cancer, 2021, 148 (1): 28-37.

4. Sopik V. International variation in breast cancer incidence and mortality in young women. Breast Cancer Res Treat, 2021, 186 (2): 497-507.

5. Riggio Al, Varley KE, Welm AL. The lingering mysteries of metastatic recurrence in breast cancer. $\mathrm{Br} \mathrm{J}$ Cancer, 2021, 124 (1): 13-26.

6. Wu Y, Han Y, Yu P, Ouyang Q, Yan M, Wang X, Hu X, Jiang Z, Huang T, Tong Z, Wang S, Yin Y, Li H, Yang R, Yang H, Teng Y, Sun T, Cai L, Li H, Chen X, He J, Liu X, Yang S, Qiao Y, Fan J, Wang J, Xu B. Endocrine Therapy for Hormone Receptor-Positive Advanced Breast Cancer: A Nation-Wide Multicenter Epidemiological Study in China. Front Oncol, 2021, 10: 599604.

7. Liang Y, Zhang H, Song X, Yang Q. Metastatic heterogeneity of breast cancer: Molecular mechanism and potential therapeutic targets. Semin Cancer Biol, 2020, 60: 14-27.

8. JAgarwal S, Kashaw SK. Potential target identification for breast cancer and screening of small molecule inhibitors: A bioinformatics approach. J Biomol Struct Dyn, 2021, 39 (6): 1975-1989.

9. Soudyab M, Iranpour M, Ghafouri-Fard S. The Role of Long Non-Coding RNAs in Breast Cancer. Arch Iran Med, 2016, 19 (7): 508-517. 
10. Yang F, Lyu S, Dong S, Liu Y, Zhang X, Wang O. Expression profile analysis of long noncoding RNA in HER-2-enriched subtype breast cancer by next-generation sequencing and bioinformatics. Onco Targets Ther, 2016, 9: 761-772.

11. Shen X, Xie B, Ma Z, Yu W, Wang W, Xu D, Yan X, Chen B, Yu L, Li J, Chen X, Ding K, Cao F. Identification of novel long non-coding RNAs in triple negative breast cancer. Oncotarget, 2015, 6 (25): 21730-21739.

12. Yang F, Lv SX, Lv L, Liu YH, Dong SY, Yao ZH, Dai XX, Zhang XH, Wang OC. Identification of IncRNA FAM83H-AS1 as a novel prognostic marker in luminal subtype breast cancer. Onco Targets Ther, 2016, 9: 7039-7045.

13. Ye N, Wang B, Quan ZF, Cao SJ, Wen XT, Huang Y, Huang XB, Wu R, Ma XP, Yan QG. Functional roles of long non-coding RNA in human breast cancer. Asian Pac J Cancer Prev, 2014, 15 (15): 5993-5997.

14. Liu B, Pan CF, Ma T, Wang J, Yao GL, Wei K, Chen YJ. Long non-coding RNA AK001796 contributes to cisplatin resistance of non-small cell lung cancer. Mol Med Rep, 2017, 16 (4): 4107-4112.

15. Ouyang W, Ren L, Liu G, Chi X, Wei H. LncRNA MIR4435-2HG predicts poor prognosis in patients with colorectal cancer. Peer J, 2019, 7: e6683.

16. Kong Q, Liang C, Jin Y, Pan Y, Tong D, Kong Q, Zhou J. The IncRNA MIR4435-2HG is upregulated in hepatocellular carcinoma and promotes cancer cell proliferation by upregulating miRNA-487a. Cell Mol Biol Lett, 2019, 24: 26.

17. Zhu L, Wang A, Gao M, Duan X, Li Z. LncRNA MIR4435-2HG triggers ovarian cancer progression by regulating miR-128-3p/CKD14 axis. Cancer Cell Int, 2020, 20: 145.

18. Chen D, Tang P, Wang Y, Wan F, Long J, Zhou J, Zhuang M, Chen X. Downregulation of long noncoding RNA MR4435-2HG suppresses brest cancer progression via the Wnt/ $\beta$-catenin signaling pathway. Oncol Lett, 1011, 21 (5): 373.

19. Luo P, Wu S, Ji K, Yuan X, Li H, Chen J, Tian Y, Qiu Y, Zhong X. LncRNA MIR4435-2HG mediates cisplatin resistance in HCT116 by regulating Nrf2 and HO-1. PLoS One, 2020, 15 (11): e0223035.

20. Nötzold L, Frank L, Gandhi M, Polycarpou-Schwarz M, Groß M, Gunkel M, Beil N, Erfle H, Harder N, Rohr K, Trendel J. Krijgsveld J, Longerich T, Schimacher P, Boutros M, Erhardt S, Diederichs S. The long-coding RNA LINC00152 is essential for cell cycle progression through nitosis in HeLa cells. Sci Rep, 2017, 7 (1): 2265.

21. Wang H, Wu M, Lu Y, He K, Cai X, Yu X, Lu J, Teng L. LncRNA MIR4435-2HG targets desmoplakin and promotes growth and metastasis of gastric cancer by activating Wnt/beta-catenin signaling. Aging (Albany NY), 2019, 11 (17): 6657-6673.

22. Zhang H, Meng H, Huang X, Tong W, Liang X, Li J, Zhang C, Chen M. IncRNA MIR4435-2HG promotes cancer cell migration and invasion in prostate carcinoma by upregulating TGF-beta1. Oncol Lett, 2019, 18 (4): 4016-4021.

23. Shen H, Sun B, Yang Y, Cai X, Bi L, Deng L, Zhang L. MIR4435-2HG regulates cancer cell behaviors in oral squamous cell carcinoma cell growth by upregulating TGF-beta1. Odontology, 2020, 108 (4): 553-559. 
24. Yang M, He X, Huang X, Wang J, He Y, Wei L. LncRNA MIR4435-2HG-mediated upregulation of TGFbeta1 promotes migration and proliferation of nonsmall cell lung cancer cells. Environ Toxicol, 2020, 35 (5): 582-590.

25. Dong X, Yang Z, Yang H, Li D, Qiu X. Long Non-coding RNA MIR4435-2HG Promotes Colorectal Cancer Proliferation and Metastasis Through miR-206/YAP1 Axis. Front Oncol, 2020, 10: 160.

26. Liu S, Qiao Z, Ma Q, Liu X, Ma X. LncRNA CYTOR and MIR4435-2HG in ovarian cancer and its relationship with clinicopathological features. Panminerva Med, 2019. doi: 10.23736/S00310808.19.03705-4.

27. Wang R, Liu L, Jiao J, Gao D. Knockdown of MIR4435-2HG Suppresses the Proliferation, Migration and Invasion of Cervical Cancer Cells via Regulating the miR-128-3p/MSI2 Axis in vitro. Cancer Manag Res, 2020, 12: 8745-8756.

28. Dykes IM, Emanueli C. Transcriptional and Post-transcriptional Gene Regulation by Long Non-coding RNA. Genomics Proteomics Bioinformatics, 2017, 5 (3): 177-186.

29. Salmena L, Poliseno L, Tay Y, Kats L, Pandolfi PP. A ceRNA hypothesis: the Rosetta Stone of a hidden RNA language? Cell, 2011,146 (3): 353-358.

30. Chan JJ, Tay Y. Noncoding RNA: RNA Regulatory Networks in Cancer. Int J Mol Sci, 2018, 19 (5): 1310.

31. Xiao Y, Bao Y, Tang L, Wang L. LncRNA MIR4435-2HG is downregulated in osteoarthritis and regulates chondrocyte cell proliferation and apoptosis. J Orthop Surg Res, 2019, 14 (1): 247.

32. Qian H, Chen L, Huang J, Wang X, Ma S, Cui F, Luo L, Ling L Luo K, Zheng G. The IncRNA MIR4435$2 \mathrm{HG}$ promotes lung cancer progression by activating $\beta$-catenin signalling. J Mol Mel (Berl), 2018, 96 (8): 753-764.

33. Xiao J, Niu S, Zhu J, Lv L, Deng H, Pan D, Shen D, Xu C, Shen Z, Tao T. miR-22-3p enhances multichemoresistance by targeting NET1 in bladder cancer cells. Oncol Rep, 2018, 39 (6): 2731-2740.

34. Zhang Z, Li M, Zhang Z. IncRNA MALAT1 modulates oxaliplatin resistance of gastric cancer via sponging miR-22-3p. Onco Targets Ther, 2020, 13: 1343-1354.

35. He W, Zhang Y, Xia S. LncRNA NNT-AS1 promotes non-small cell lung cancer progression through regulating miR-22-3p/YAP1 axis. Thorac Cancer, 2020, 11 (3): 549-560.

36. Gan L, Lv L, Liao S. Long non-coding RNA H19 regulates cell growth and metastasis via the miR-223p/Snail1 axis in gastric cancer. Int J Oncol, 2019, 54 (6): 2157-2168.

37. Loh HY, Norman BP, Lai KS, Rahman NMANA, Alitheen NBM, Osman MA. The Regulatory Role of MicroRNAs in Breast Cancer. Int J Mol Sci, 2019, 20 (19): 4940.

38. Rovillain E, Mansfield L, Lord CJ, Ashworth A, Jat PS. An RNA interference screen for identifying downstream effectors of the p53 and pRB tumour suppressor pathways involved in senescence. BMC Genomics, 2011, 12: 355.

39. Dodeller F, Gottar M, Huesken D, lourgenko V, Cenni B. The lysosomal transmembrane protein $9 B$ regulates the activity of inflammatory signaling pathways. J Biol Chem, 2008 , 283 (31): 21487- 
Figures
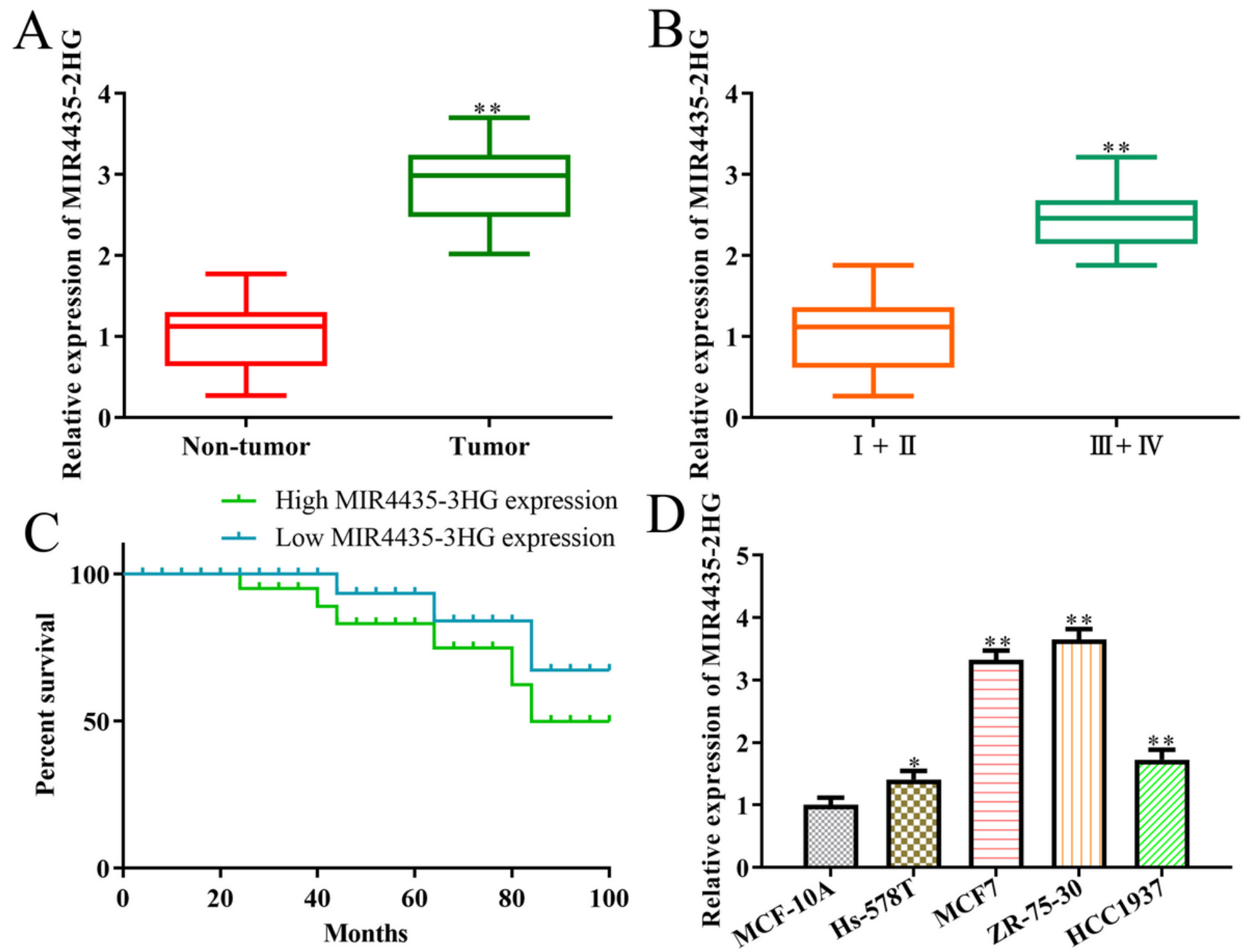

Figure 1

MIR4435-2HG is highly expressed in breast cancer tissues and cell lines. (A) The expression of MIR4435$2 \mathrm{HG}$ in breast cancer tissues was detected by qRT-PCR. ${ }^{\star *} \mathrm{P}<0.01$ vs. non-tumor tissues. (B) The expression of MIR4435-2HG in breast cancer tissues at the different stages was detected by qRT-PCR. **P $<0.01$ vs. tumor tissues at stage I+II. (C) Patients with low MIR4435-2HG levels had longer survival times compared with patients with high MIR4435-2HG expression. (D) The expression of MIR4435-2HG in breast cancer cell lines was detected using qRT-qPCR. ${ }^{*} P<0.05,{ }^{\star *} \mathrm{P}<0.01, * \star * P<0.001$ vs. MCF-10A cells. 
A

B $\quad$ MCF
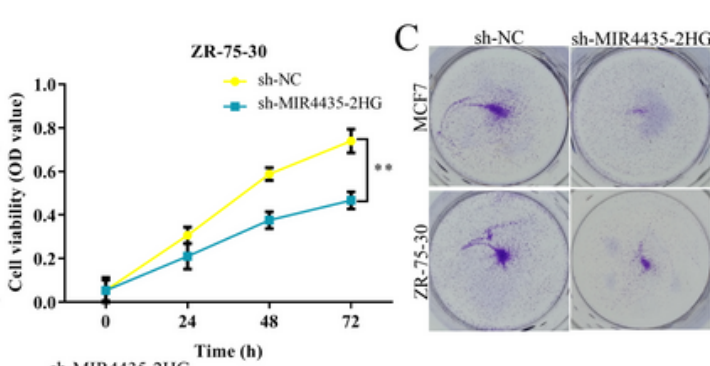

$\mathrm{D}$

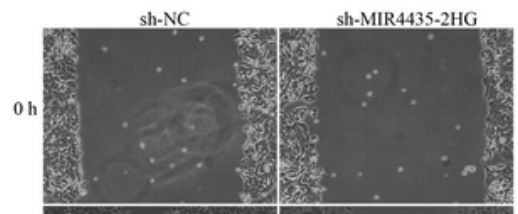

Time (h) sh-MIR4435-2HG Time (h)
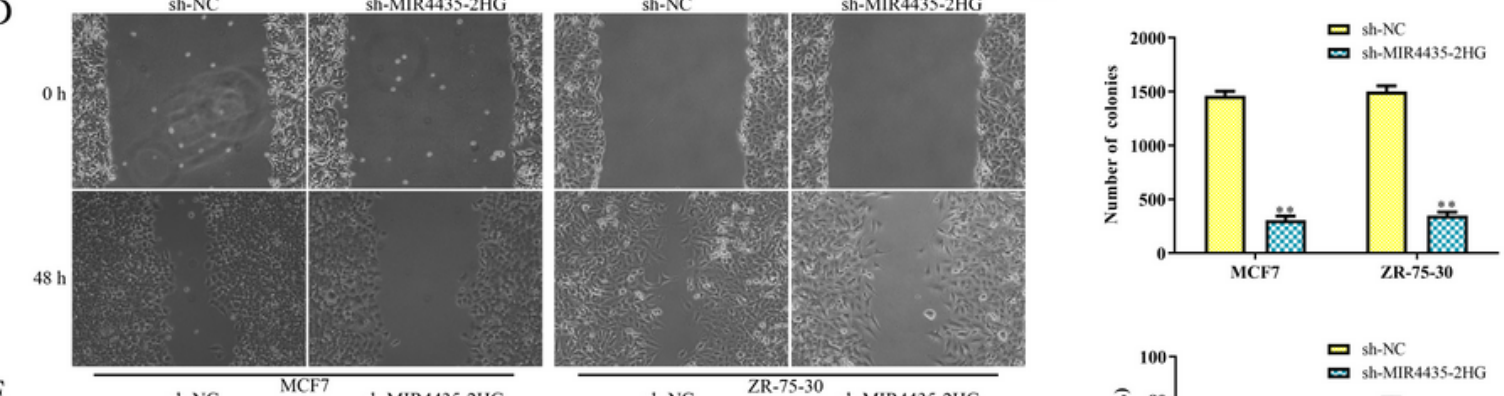

E
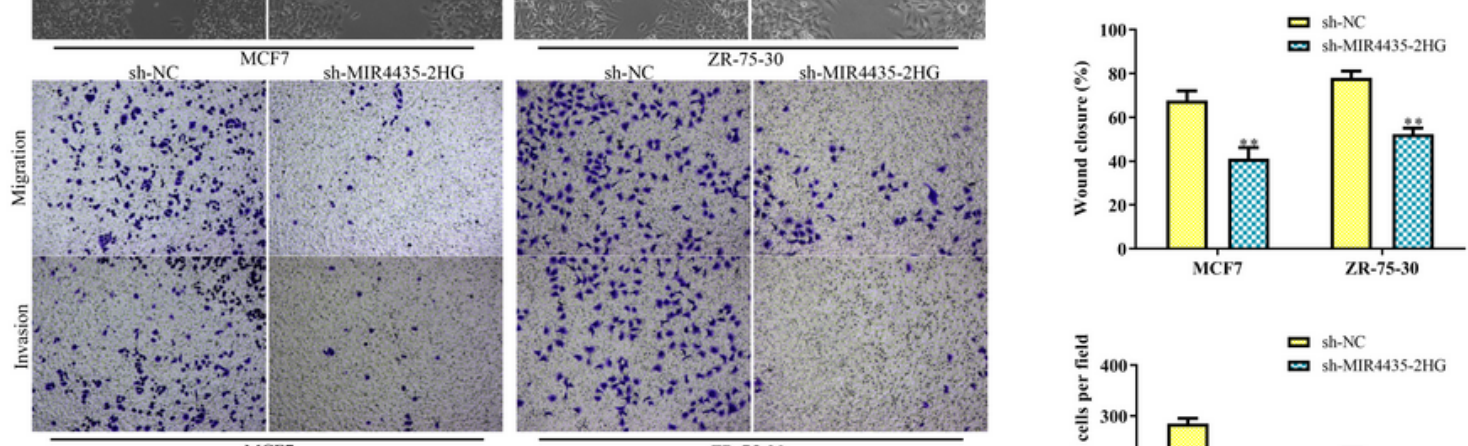

F
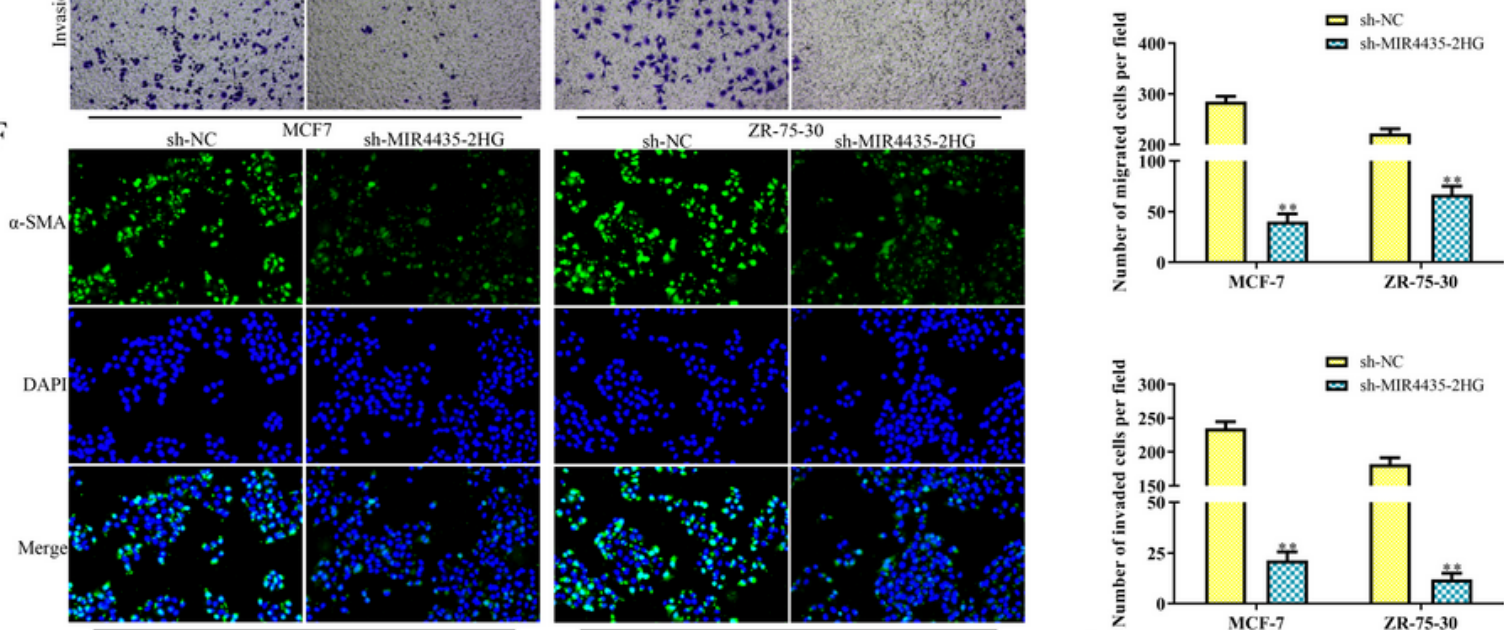

G
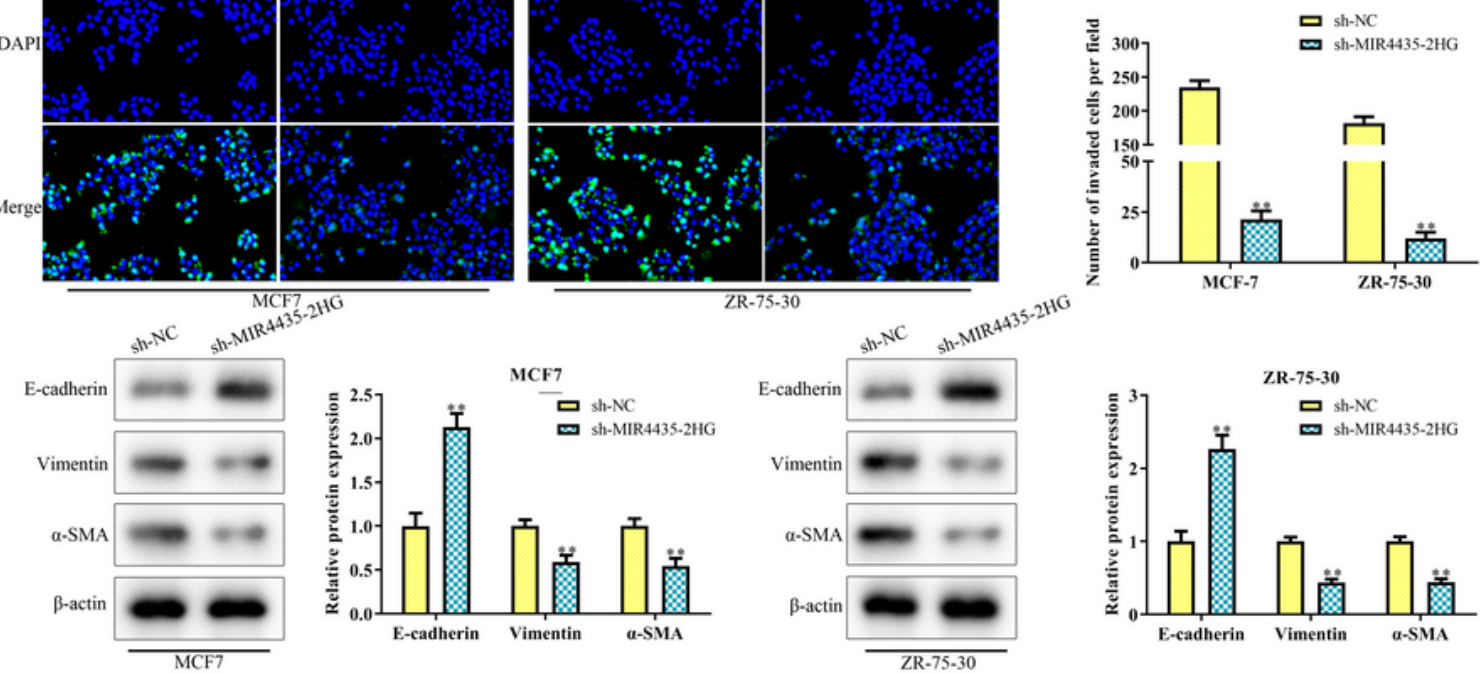

Figure 2

Down-regulation of MIR4435-2H inhibits the viability, proliferation, migration, invasion and EMT of breast cancer cells. (A) The expression of MIR4435-2H in MCF-7 and ZR-75-30 cells transfected with shMIR4435-2H was determined by qRT-qPCR. (B) The viability of MCF-7 and ZR-75-30 cells transfected with sh-MIR4435-2H was assessed by CCK-8 assay at the indicated times. (C) The proliferation of MCF-7 and ZR-75-30 cells transfected with sh-MIR4435-2H was assessed by colony formation assay. (D) The 
migration of MCF-7 and ZR-75-30 cells transfected with sh-MIR4435-2H was determined by wound healing assay. (E) The migration and invasion of MCF-7 and ZR-75-30 cells transfected with sh-MIR4435$2 \mathrm{H}$ were determined by transwell migration and invasion assays. $(\mathrm{F})$ The protein expression of a-SMA in MCF-7 and ZR-75-30 cells transfected with sh-MIR4435-2H was assessed by immunofluorescence assay.

(G) The expression levels of proteins, including E-cadherin, vimentin and a-SMA, in MCF-7 and ZR-75-30 cells transfected with sh-MIR4435-2H were evaluated by western blot assay. ${ }^{\star \star} P<0.01,{ }^{\star \star \star} P<0.001$ vs. sh-NC.

A

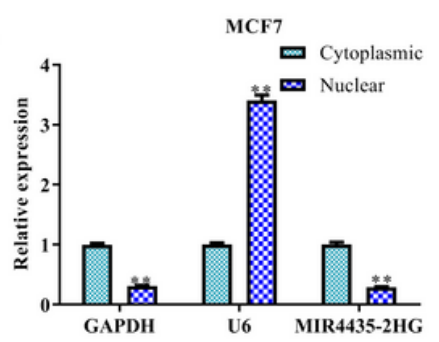

$\mathrm{D}$
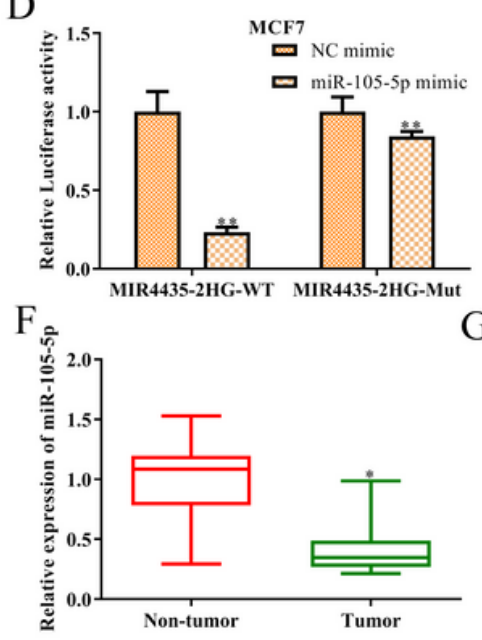
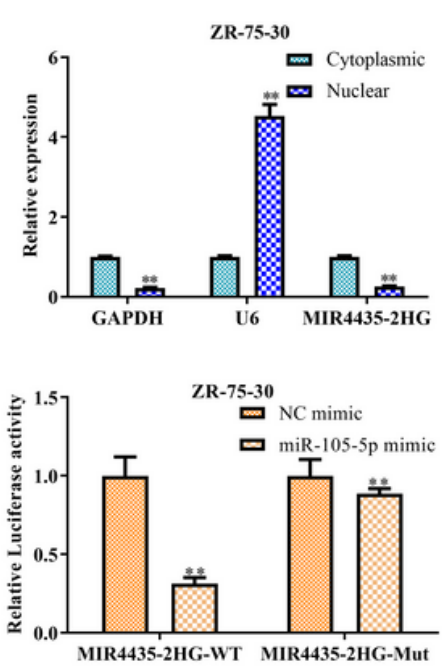

$\mathrm{G}$

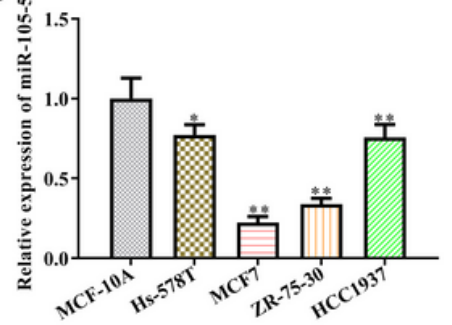

$\mathrm{B}$
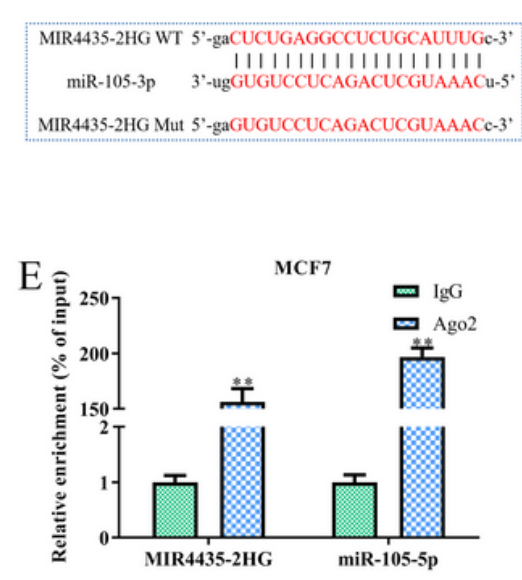

$\mathrm{H}$

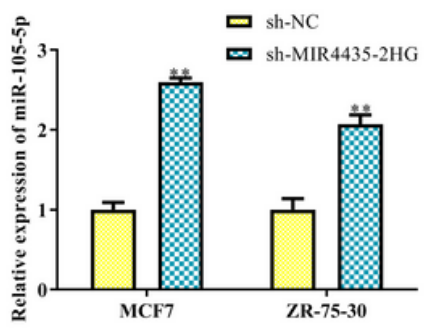

C
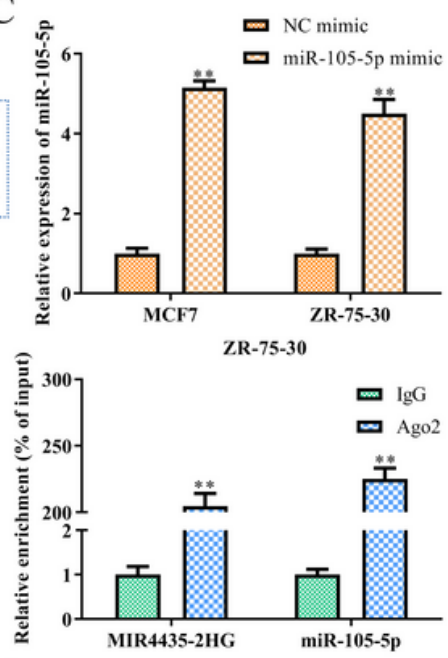

$I$ के

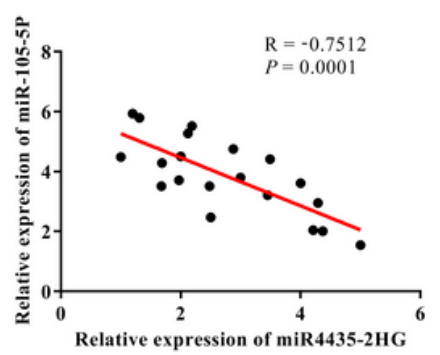

\section{Figure 3}

MIR4435-2H acts as a ceRNA via sponging miR-22-3p. (A) The cellular location of MIR4435-2H was assessed by subcellular fractionation analysis. $* \star \star P<0.001$ vs. cytoplasmic fraction. (B) Binding sites between MIR4435-2H and miR-22-3p. (C) The expression of miR-22-3p in MCF-7 and ZR-75-30 cells transfected with miR-22-3p mimic was detected by the qRT-PCR assay. (D) The interaction between

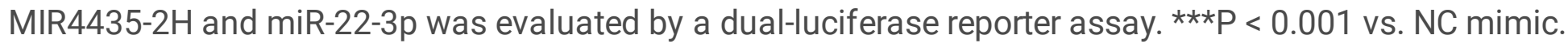
(E) Relative enrichment of MIR4435-2H and miR-22-3p in MCF-7 and ZR-75-30 cells was detected by RNA pull-down assay. ${ }^{\star \star *} \mathrm{P}<0.001 \mathrm{vs}$. IgG. The expression of miR-22-3p in $(\mathrm{F})$ breast cancer tissues and $(\mathrm{G})$ cell lines was detected by the qRT-PCR assay. ${ }^{*} P<0.05$ vs. non-tumor tissues, ${ }^{*} P<0.05$, ${ }^{\star \star \star} P<0.001$ vs. MCF-10A cells. $(\mathrm{H})$ The expression of miR-22-3p in MCF-7 and ZR-75-30 cells transfected with shMIR4435-2H was assessed by qRT-PCR assay. ${ }^{\star \star \star} \mathrm{P}<0.001$ vs. sh-NC. (I) Correlation analysis between MIR4435-2H and miR-22-3p in breast cancer tissues. 


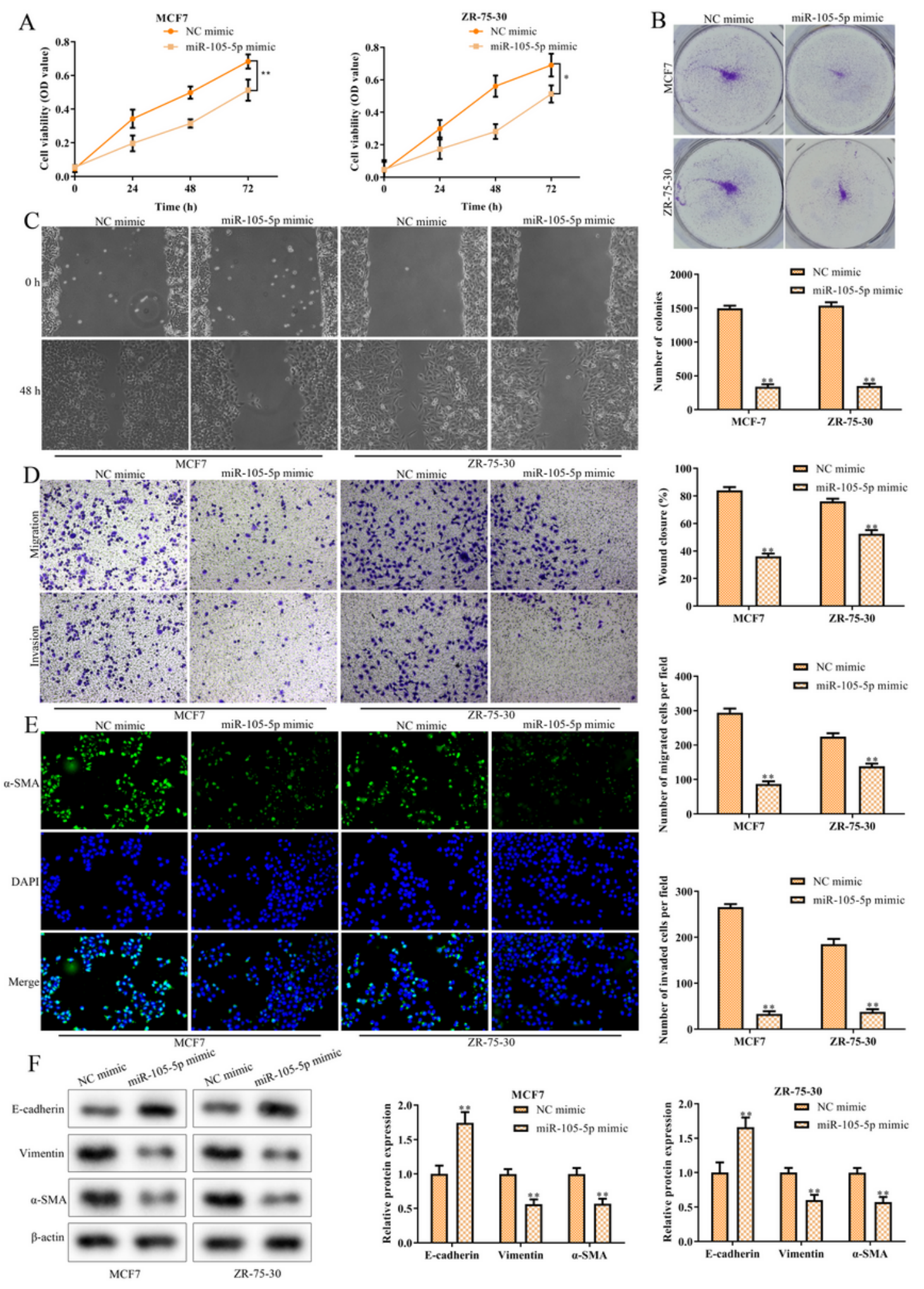

Figure 4

Up-regulation of miR-22-3p inhibits the viability, proliferation, migration, invasion and EMT of breast cancer cells. (A) The viability of MCF-7 and ZR-75-30 cells transfected with miR-22-3p mimic was assessed by CCK-8 assay at the indicated times. (B) The proliferation of MCF-7 and ZR-75-30 cells transfected with miR-22-3p mimic was assessed by colony formation assay. (C) The migration of MCF-7 and ZR-75-30 cells transfected with miR-22-3p mimic was determined by wound healing assay. (D) The 
migration and invasion of MCF-7 and ZR-75-30 cells transfected with miR-22-3p mimic were determined by transwell migration and invasion assays. (E) The protein expression of a-SMA in MCF-7 and ZR-75-30 cells transfected with miR-22-3p mimic was assessed by immunofluorescence assay. $(F)$ The expression levels of proteins, including E-cadherin, vimentin and a-SMA, in MCF-7 and ZR-75-30 cells transfected with miR-22-3p mimic were evaluated by western blot assay. ${ }^{*} P<0.05,{ }^{*} P<0.01,{ }^{* \star *} P<0.001$ vs. NC mimic.

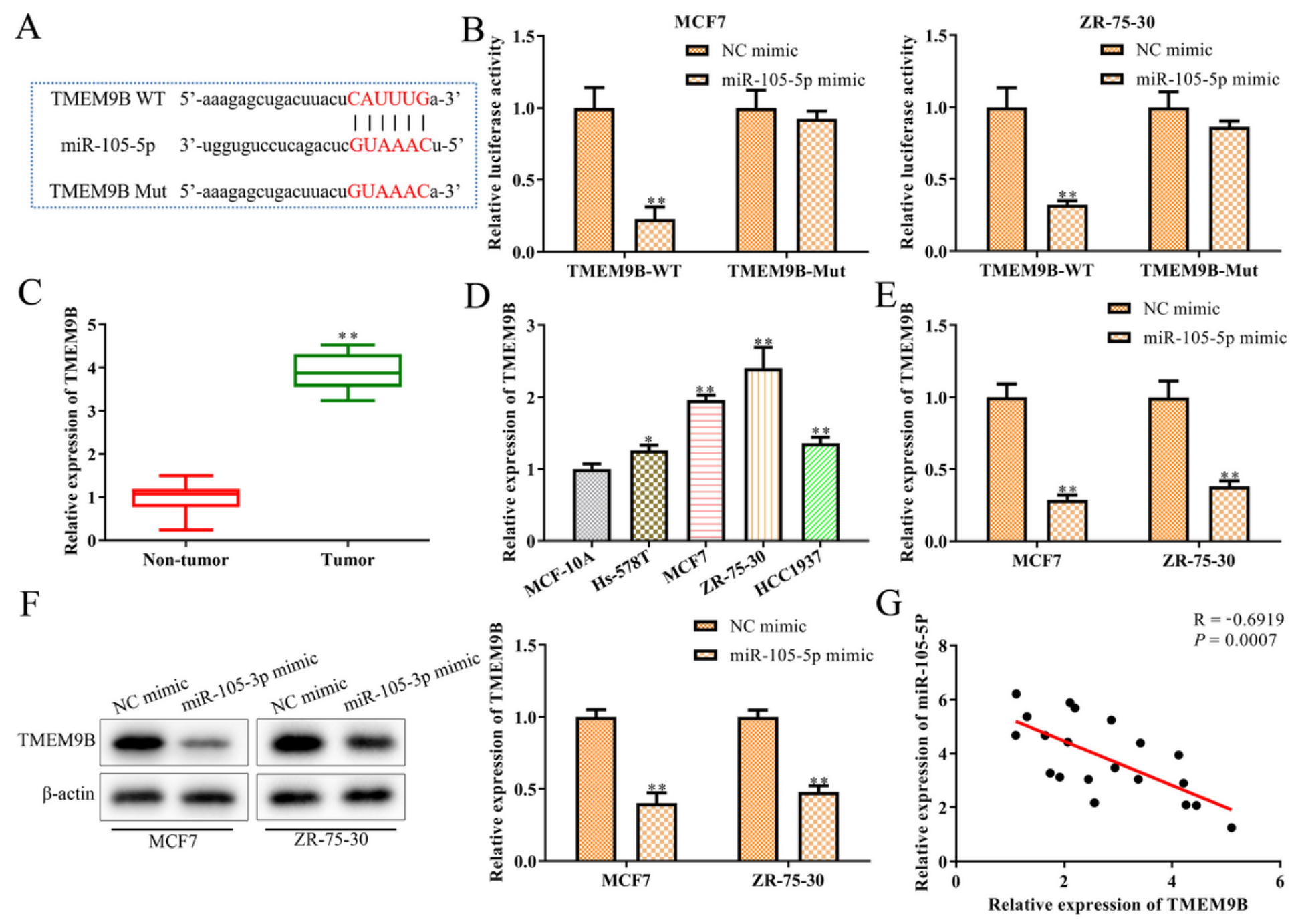

Figure 5

TMEM9B is a direct target gene of miR-22-3p and is negatively associated with miR-22-3p. (A) Binding sites between miR-22-3p and TMEM9B. (B)The interaction between miR-22-3p and TMEM9B was evaluated by a dual-luciferase reporter assay. ${ }^{* *} \mathrm{P}<0.001 \mathrm{vs}$. NC mimic. The expression of TMEM9B in (C) breast cancer tissues and (D) cell lines was detected by the qRT-PCR assay. ${ }^{*} P<0.01$ vs. non-tumor tissues, ${ }^{*} P<0.05,{ }^{*} P<0.01$ vs. MCF-10A cells. The $(E)$ mRNA and $(F)$ protein levels of TMEM9B in MCF7 and ZR-75-30 cells transfected with miR-22-3p mimic were assessed by qRT-PCR and western blot assays. ${ }^{* \star *} \mathrm{P}<0.001$ vs. NC mimic. (G) Correlation analysis between miR-22-3p and TMEM9B in breast cancer tissues. 


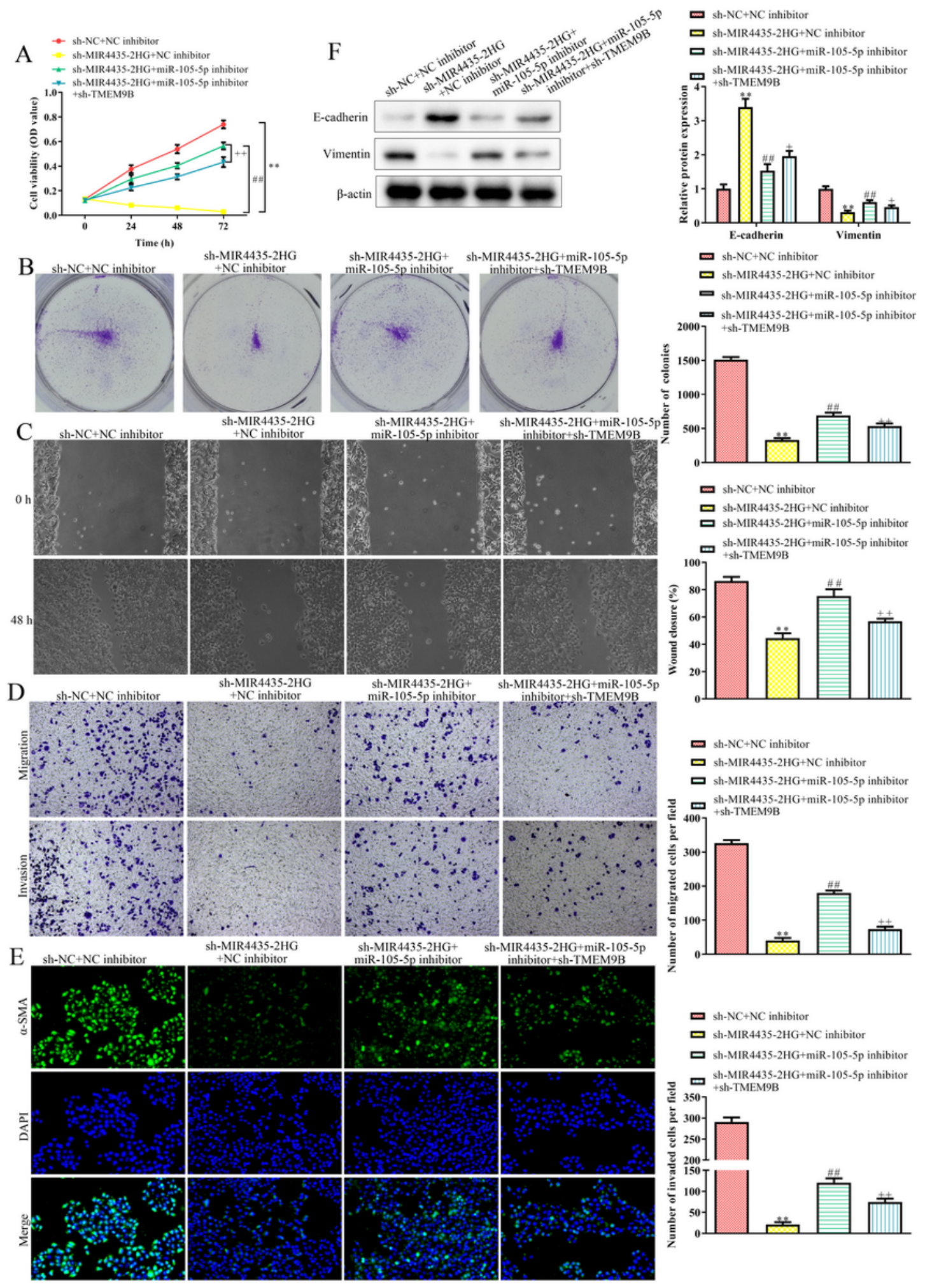

Figure 6

MIR4435-2H regulates the viability, proliferation, migration, invasion and EMT of breast cancer cells by modulating the miR-22-3p/TMEM9B axis. (A) The viability of MCF-7 and ZR-75-30 cells after transfection was assessed by CCK-8 assay at the indicated times. (B) The proliferation of MCF-7 and ZR-75-30 cells after transfection was assessed by colony formation assay. (C) The migration of MCF-7 and ZR-75-30 cells after transfection was determined by wound healing assay. (D) The migration and invasion of MCF- 
7 and ZR-75-30 cells after transfection were determined by transwell migration and invasion assays. (E) The protein expression of a-SMA in MCF-7 and ZR-75-30 cells after transfection was assessed by immunofluorescence assay. $(F)$ The expression levels of proteins, including E-cadherin, vimentin and aSMA, in MCF-7 and ZR-75-30 cells after transfection were evaluated by western blot assay. ${ }^{*} \mathrm{P}<0.01$ vs. sh-NC+NC inhibitor, \#\#P<0.01, \#\#\#P<0.001 vs. sh-MIR4435-2H+NC inhibitor, $\$ \mathrm{P}<0.05$,

$$
P<0.01,
$$

$\$ \mathrm{P}<0.001$ vs. sh-MIR4435-2H+miR-22-3p inhibitor. 